\title{
Uniqueness of One-Dimensional Continuum Gibbs States
}

\author{
David Klein \\ Department of Mathematics, Louisiana State University, Baton Rouge, LA 70803, USA
}

\begin{abstract}
We investigate one-dimensional continuum grandcanonical Gibbs states corresponding to finite range superstable many-body potentials. Absence of phase transitions in the sense of uniqueness of the tempered Gibbs state is proved for potentials without hard-core by first proving uniqueness of the Gibbs measures for related hard-core potentials and then taking an appropriate limit of those Gibbs measures.
\end{abstract}

\section{Introduction}

The absence of phase transitions has been established for a wide variety of onedimensional statistical mechanical models. The majority of papers on this subject have focused on lattice or hard-core continuum systems, due to the technical difficulties which arise in continuum models without hard-core restrictions. For a brief survey of results of this type we refer the reader to the introduction of Dobrushin's paper [1]. More recently, results have been obtained for the nonhardcore continuum case. Campanino, Capocaccia, and Olivieri in [2] proved the analyticity of the infinite volume pressure as a function of the interaction parameters for superstable, two-body, slowly decaying potentials (without hardcore) via renormalization group techniques. Suhov [7] proved the uniqueness of the Gibbs state, and related properties, for positive pair potentials with exponential decay, and indicated extensions of his results to other classes of potentials.

In this paper we prove, by different methods, uniqueness of the tempered Gibbs state for finite range, superstable, many-body interactions without hard-core for one-dimensional systems. The finite range restriction does not seem to be crucial to the method of proof, but significantly simplifies the arguments, which we believe may have applications to higher dimensional models.

For a given potential $V$ without hard-core, we prove in Sect. 2 uniqueness of the Gibbs state corresponding to the perturbed potential $V+\varphi_{N}$ for $N=2,3,4, \ldots$, where $\varphi_{N}=\infty$ for configurations with more than $N$ particles in any interval of length 1 , and $\varphi_{N}=0$ otherwise (see Definition 1.1). The method of proof is based on 
a technique of Dobrushin [1] developed for lattice and hard-core models. Dobrushin's results establish uniqueness for the Gibbs state corresponding to $V+\varphi_{N}$ when $N=2$, since in this case $V+\varphi_{N}$ is a hard-core potential in the usual sense. When $N$ is extremely large so that, for example, $N$ particles in an interval of length 1 corresponds to a density which greatly exceeds that of any known form of matter, one would not expect physically significant differences in the behavior of systems governed respectively by $V$ and $V+\varphi_{N}$. A version of this is in fact established in Sect. 3 where we prove that any extremal tempered Gibbs state for $V$ is a limit, as $N \rightarrow \infty$, of extremal tempered Gibbs states for $V+\varphi_{N}$. Uniqueness is then easily proved.

We remark that perturbed potentials of the form $V+\varphi_{N}$ along with the corresponding Gibbs states and correlation functions were studied for higher dimensional models in $[3,4]$.

\section{Notation and Definitions}

Let $X(\Lambda)$ denote the set of all locally finite subsets (configurations) of the Borel subset $\Lambda$ of the real line $\mathbb{R}$. Let $B_{\Lambda}$ be the $\sigma$-field on $X(\Lambda)$ generated by all sets of the form $\{s \in X(\Lambda):|s \cap B|=m\}$, where $B$ runs over all bounded Borel subsets of $\Lambda, m$ runs over the set of nonnegative integers, and $|\cdot|$ denotes cardinality. As in [3-5] we make the natural identification

$$
\left(X(\mathbb{R}), B_{\mathbb{R}}\right)=\prod_{i=-\infty}^{\infty}\left(X((i, i+1]), B_{(i, i+1]}\right) .
$$

Let $X_{F}$ denote the set of configurations in $X(\mathbb{R})$ of finite cardinality, and $X_{N}(\Lambda)$ the set of configurations in $X(\Lambda)$ of cardinality $N$.

For a bounded Borel set $\Lambda$, let $T: \Lambda^{N} \rightarrow X_{N}(\Lambda)$ be the map which takes the ordered $N$-tuple $\left(x_{1}, \ldots, x_{N}\right)$ to the unordered set $\left\{x_{1}, \ldots, x_{N}\right\}$. In a natural way $T$ defines an equivalence relation on $\Lambda^{N}$, and $X_{N}(\Lambda)$ may be regarded as the set of equivalence classes induced by $T$. For $n=1,2,3, \ldots$, let $d^{n} x$ be the projection of $n$-dimensional Lebesgue measure onto $X_{N}(\Lambda)$ under the map $T$. The measure $d^{0} x$ assigns mass 1 to $X_{0}(\Lambda)=\{\emptyset\}$. Define as in $[3,4,5]$

$$
v_{\Lambda}(d x)=\sum_{n=0}^{\infty} \frac{z^{n}}{n !} d^{n} x
$$

where $z$ is chemical activity. The measure $v_{\Lambda}$, when normalized to a probability measure, is the finite volume Gibbs state for the zero potential in $\Lambda$.

We will consider $B_{\mathbb{R}}$-measurable many-body potentials $V: X_{F} \rightarrow(-\infty,+\infty]$ of the form

$$
V(x)=\sum_{N=1}^{\infty} \sum_{\substack{y \subset x \\|y|=N}} \phi_{N}(y),
$$

where the function $\phi_{N}: X_{N}(\mathbb{R}) \rightarrow(-\infty,+\infty]$ is called an $N$-body potential. For later reference we list some restrictions to be imposed on a potential $V$. 
Condition 1.1. a) $V$ is superstable (see [6]).

b) $\inf \left\{\phi_{N}(y): y \in X_{F},|y|=N, N \geqq 1\right\}>-\infty$, where $\phi_{N}$ is related to $V$ as in (1.3).

c) $V$ has finite range, i.e., there exists $R_{0}>0$ such that if

$$
\max _{i j}\left|x_{i}-x_{j}\right|>R_{0} \text {, }
$$

then $\phi_{N}\left(x_{1}, \ldots, x_{N}\right)=0$, for all $N \geqq 2$.

Definition 1.1. For a configuration $x=\left(x_{1}, \ldots, x_{N}\right) \in X_{N}(\mathbb{R})$, let

$$
\varphi_{N}(x)= \begin{cases}\infty & \text { if } \max _{i j}\left|x_{i}-x_{j}\right|<1 \\ 0 & \text { otherwise. }\end{cases}
$$

For a given potential $V$, let

$$
V^{N}=V+\varphi_{N} .
$$

As in [5] we define the $B_{\mathbb{R}}$-measurable set $R_{\Lambda} \subset X(\mathbb{R})$ so that $V(x \mid s)$ represents the energy of the configuration $x \in X(\Lambda)$, assuming the configuration $s \in R_{\Lambda} \cap X\left(\Lambda^{c}\right)$. The finite volume Gibbs state $\mu_{\Lambda}(d x \mid s)$ for the bounded Borel set $\Lambda$ (with positive Lebesgue measure), potential $V$, inverse temperature $\beta$, chemical activity $z$, and external configuration $s \in R_{\Lambda} \cap X\left(\Lambda^{c}\right)$ is given by

$$
\mu_{\Lambda}(d x \mid s)=\frac{\exp [-\beta V(x \mid s)]}{Z_{\Lambda}(s)} v_{\Lambda}(d x),
$$

where the constant $Z_{\Lambda}(s)$ makes $\mu_{\Lambda}(d x \mid s)$ a probability measure. If $V$ satisfies Condition 1.1, then $1 \leqq Z_{A}(s)<\infty$. If $s \notin R_{\Lambda}$, define $\mu_{\Lambda}(d x \mid s)$ to be the zero measure.

Let $\left\{\pi_{\Lambda}\right\}$ denote the specification associated with $\beta, z$, and $V$ (see $[5$, p. 16]) defined by

$$
\pi_{\Lambda}(A, s)=\int_{A^{\prime}} \mu_{\Lambda}\left(d x \mid s \cap \Lambda^{c}\right)
$$

where $A \in B_{\mathbb{R}}, A^{\prime}=\left\{x \in X(\Lambda): x \cup\left(s \cap \Lambda^{c}\right) \in A\right\}$, and $s \in X(\mathbb{R})$.

The following definition comes from Preston [5].

Definition 1.2. For positive integers $n$ and $m$, let $I_{n}=[-2 n,+2 n] \subset \mathbb{R}$ and

$$
U_{n m}=\left\{s \in X(\mathbb{R}):\left|s \cap\left(I_{n} \backslash I_{n-1}\right)\right| \leqq 4 m\right\} .
$$

For a given potential $V$, let

$$
D=\{s \in X(\mathbb{R}): V(y)<\infty \text { for all } y \subset s \text { with }|y|<\infty\},
$$

and define

$$
U_{m}=D \bigcap_{n \geqq 1} U_{n m}, \text { and } U_{\infty}=\bigcup_{m \geqq 1} U_{m} .
$$

When $V$ is specified, let $U_{\infty}^{N}$ correspond to the potential $V^{N}$ given in Definition 1.1.

Definition 2.3. A probability measure $\sigma$ on $\left(X(\mathbb{R}), B_{\mathbb{R}}\right)$ is a Gibbs state for the specification $\left\{\pi_{A}\right\}$ if

$$
\sigma\left(\pi_{\Lambda}(A, s)\right)=\sigma(A)
$$

for every $A \in B_{\mathbb{R}}$ and every bounded Borel set $\Lambda \subset \mathbb{R}$. If in addition, $\sigma\left(U_{\infty}\right)=1$, then $\sigma$ is a tempered Gibbs state. 
Definition 2.4. For a potential $V$, Borel sets $\Lambda \subset \tilde{\Lambda}$ with positive Lebesgue measures, and $s \in U_{\infty}$, the finite volume Gibbs density $r_{\tilde{\Lambda}}^{\Lambda}(x \mid s)$ is given by

$$
r_{\tilde{\Lambda}}^{\Lambda}(x \mid s)=\int_{X(\tilde{\Lambda} \backslash \Lambda)} \frac{\exp \left[-\beta V\left(x \cup y \mid s \cap \tilde{\Lambda}^{c}\right)\right]}{Z_{\tilde{\Lambda}}\left(s \cap \tilde{\Lambda}^{c}\right)} v_{\tilde{\Lambda} \backslash \Lambda}(d y) .
$$

Definition 2.5. A function $f$ on $X(\mathbb{R})$ is a cylinder function if $f(s)=f(s \cap \Lambda)$ for some bounded set $\Lambda \subset \mathbb{R}$ and all $s \in X(\mathbb{R})$. A subset $A \subset X(\mathbb{R})$ is a cylinder set if the characteristic function for $A$ is a cylinder function.

Note that if $f$ is a $B_{\Lambda}$-measurable function on $X(\Lambda)$, then we may regard $f$ as a $B_{\mathbb{R}}$-measurable cylinder function on $X(\mathbb{B})$ by defining $f(s)=f(s \cap \Lambda)$ for $s \in X(\mathbb{R})$. In this case,

$$
\pi_{\tilde{\Lambda}}(f \mid s) \equiv \int f(x) \pi_{\tilde{\Lambda}}(d x \mid s)=\int_{x(\Lambda)} f(x) r_{\tilde{\Lambda}}^{\Lambda}(x \mid s) v_{\Lambda}(d x)
$$

\section{Hard-Core Case}

In this section we prove, for any $\beta$ and $z$, uniqueness of the Gibbs state $\sigma_{N}$ corresponding to the potential $V^{N}$ (see Definition 1.1) when $V$ satisfies Condition 1.1. The method of proof relies on techniques developed by Dobrushin in [1]. We note that with Condition 1.1, existence of a Gibbs state for $V^{N}$ was proved in [4] (see also [5,6]), and it is not difficult to see that any Gibbs state for $V^{N}$ is tempered.

The strategy for uniqueness is to prove that for any $B_{\mathbb{R}}$-measurable cylinder function $f$ on $X(\mathbb{R})$,

$$
\lim _{n \rightarrow \infty} \sup _{s, t \in U_{\infty}^{N}}\left|\pi_{[-n, n]}^{N}(f, s)-\pi_{[-n, n]}^{N}(f, t)\right|=0,
$$

where $\left\{\pi_{\Lambda}^{N}\right\}$ is the specification for $V^{N}, \beta, z$ defined by (1.6). Uniqueness of the Gibbs state then follows from [5, Lemma 9.3].

Let $\left(X, B_{X}\right)$ be a measurable space and let $\mu_{1}$ and $\mu_{2}$ be probability measures on $\left(X, B_{X}\right)$. The variation distance between the measures $\mu_{1}$ and $\mu_{2}$ is defined as

$$
\varrho\left(\mu_{1}, \mu_{2}\right)=\sup _{A \in B_{X}}\left|\mu_{1}(A)-\mu_{2}(A)\right| \text {. }
$$

If $\mu_{1}$ and $\mu_{2}$ have respective densities $p_{1}$ and $p_{2}$ with respect to a finite measure $v$ on $X$, then defining $\varrho\left(p_{1}, p_{2}\right)=\varrho\left(\mu_{1}, \mu_{2}\right)$, we have

$$
\varrho\left(p_{1}, p_{2}\right)=1 / 2 \int_{X}\left|p_{1}(x)-p_{2}(x)\right| v(d x)=1-\int_{X} \min \left(p_{1}(x), p_{2}(x)\right) v(d x) .
$$

Suppose, now that $f$ is a bounded $B_{\mathbb{R}}$-measurable cylinder function with $f(x)=f(x \cap J)$, where $J$ is an interval on $\mathbb{R}$. Then for $s, t \in U_{\infty}^{N}$ and any interval $I \supset J$,

$$
\begin{aligned}
\left|\pi_{I}(f, s)-\pi_{I}(f, t)\right| & =\left|\int_{X(J)} f(x)\left[r_{I}^{J}(x \mid s)-r_{I}^{J}(x \mid t)\right] v_{J}(d x)\right| \\
& \leqq\|f\|_{\infty} \int_{X(J)}\left|r_{I}^{J}(x \mid s)-r_{I}^{J}(x \mid t)\right| v_{J}(d x) \\
& \leqq 2\|f\|_{\infty} \varrho\left(r_{I}^{J}(\cdot \mid s), r_{I}^{J}(\cdot \mid t)\right)
\end{aligned}
$$


From (2.4) we see that (2.1) holds for any bounded measurable cylinder function provided

$$
\lim _{n \rightarrow \infty} \sup _{s, t \in U_{\infty}^{N}} \varrho\left(r_{[-n, n]}^{J}(\cdot \mid s), r_{[-n, n]}^{J}(\cdot \mid t)\right)=0
$$

for all sufficiently large finite intervals $J \subset \mathbb{R}$.

The following lemma was proved by Dobrushin in [1].

Lemma 2.1 (Dobrushin). Let $\left(X_{j}, B_{j}, v_{j}\right)$ be a measure space for $j=1,2,3$ and let

$$
\left(X, B_{X}, v\right)=\prod_{j=1}^{3}\left(X_{j}, B_{j}, v_{j}\right)
$$

be the product measure space with measure $v=v_{1} \times v_{2} \times v_{3}$. Let $p^{1}(\cdot)$ and $p^{2}(\cdot)$ be densities with respect to $v$ for probability measures on $\left(X, B_{X}\right)$. Consider the marginal densities

$$
\begin{gathered}
p_{1}^{i}\left(x_{1}\right)=\iint p^{i}\left(x_{1}, x_{2}, x_{3}\right) v_{2}\left(d x_{2}\right) v_{3}\left(d x_{3}\right), \\
p_{1,2}^{i}\left(x_{1}, x_{2}\right)=\int p^{i}\left(x_{1}, x_{2}, x_{3}\right) v_{3}\left(d x_{3}\right), \text { for } \quad i=1,2,
\end{gathered}
$$

and the similarly defined densities $p_{2}^{i}\left(x_{2}\right), p_{3}^{i}\left(x_{3}\right), p_{1,3}^{i}\left(x_{1}, x_{3}\right)$, and $p_{2,3}^{i}\left(x_{2}, x_{3}\right)$ for $i=1,2$. Suppose there exist conditional densities $p_{1}^{i}\left(x_{1} \mid x_{2}, x_{3}\right)$ and $p_{1 / 2}^{i}\left(x_{1} \mid x_{2}\right)$ for which

$$
\begin{gathered}
p^{i}\left(x_{1}, x_{2}, x_{3}\right)=p_{1}^{i}\left(x_{1} \mid x_{2}, x_{3}\right) p_{2,3}^{i}\left(x_{2}, x_{3}\right), \\
p_{1,2}^{i}\left(x_{1}, x_{2}\right)=p_{1 / 2}^{i}\left(x_{1} \mid x_{2}\right) p_{2}^{i}\left(x_{2}\right) \quad(i=1,2) .
\end{gathered}
$$

Then

$$
\varrho\left(p_{1}^{1}, p_{1}^{2}\right) \leqq \alpha_{0} \varrho\left(p_{2}^{1}, p_{2}^{2}\right)+\bar{\alpha}_{0}\left(1-\varrho\left(p_{2}^{1}, p_{2}^{2}\right)\right),
$$

where

$$
\begin{aligned}
& \alpha_{0}=\sup _{\substack{x_{j}, \tilde{x}_{j} \in X_{j} \\
j=1,2}} \varrho\left(p_{1}^{1}\left(\cdot \mid x_{2}, x_{3}\right), p_{1}^{2}\left(\cdot \mid \tilde{x}_{2}, \tilde{x}_{3}\right)\right), \\
& \bar{\alpha}_{0}=\sup _{\substack{x_{2} \in X_{2} \\
x_{3}, \tilde{x}_{3} \in X_{3}}} \varrho\left(p_{1}^{1}\left(\cdot \mid x_{2}, x_{3}\right), p_{1}^{2}\left(\cdot \mid x_{2}, \tilde{x}_{3}\right)\right) .
\end{aligned}
$$

Most of the remainder of this section is devoted to using Lemma 2.1 to establish (2.5) and (2.1).

Let $f$ be a bounded measurable cylinder function satisfying $f(x)=f(x \cap J)$, and let the interval $J=(b, c]$ be chosen so that the range of the potential $V^{N}$ is less than $c-b$. Let $s_{1}, s_{2} \in U_{\infty}^{N}$ be given and satisfy $s_{1} \cap(c, \infty)=s_{2} \cap(c, \infty)$. Define $a=2 b-c$ so that the interval $(a, b]$ has the same length as $(b, c]$. Assume that $n$ is large enough so that $[-n, n] \supset(a, b] \cup(b, c]$. In the language of Dobrushin's lemma we make the following identifications:

$$
\begin{aligned}
& \left(X_{1}, B_{1}\right)=\left(X((b, c]), B_{(b, c]}\right), \\
& \left(X_{2}, B_{2}\right)=\left(X((a, b]), B_{(a, b]}\right), \\
& \left(X_{3}, B_{3}\right)\left(X([-n, a]), B_{[-n, a]}\right) .
\end{aligned}
$$

Given a configuration $x \in X(\mathbb{R})$, let $x_{1}=x \cap(b, c], x_{2}=x \cap(a, b], x_{3}=x \cap[-n, a]$, and

$$
v=v_{[-n, a]} \times v_{(a, b]} \times v_{(b, c]} \text {. }
$$

For $i=1,2$, let

$$
p^{i}\left(x_{1}, x_{2}, x_{3}\right)=r_{[-n, n]}^{[-n, c]}\left(x_{1}, x_{2}, x_{3} \mid s_{i}\right) .
$$


It follows as in [1] that for $i=1,2$,

$$
\begin{gathered}
p_{1}^{i}\left(x_{1}\right)=r_{[-n, n]}^{(b, c]}\left(x_{1} \mid s_{i}\right), \\
p_{2}^{i}\left(x_{2}\right)=r_{[-n, n]}^{(a, b]}\left(x_{2} \mid s_{i}\right), \\
p_{1}^{i}\left(x_{1} \mid x_{2}, x_{3}\right)=r_{(b, n]}^{(b, c]}\left(x_{1} \mid x_{2} \cup x_{3} \cup\left(s_{i} \backslash[-n, b]\right)\right) .
\end{gathered}
$$

Remark 2.1. For the probability densities just defined, the conclusion of Lemma 2.1 can be modified, with no change in Dobrushin's proof, so that

where

$$
\varrho\left(p_{1}^{1}, p_{1}^{2}\right) \leqq \alpha \varrho\left(p_{2}^{1}, p_{2}^{2}\right)+\bar{\alpha}\left[1-\varrho\left(p_{2}^{1}, p_{2}^{2}\right)\right],
$$

and

$$
\begin{gathered}
\alpha=\sup \left\{\varrho\left(p_{1}^{1}\left(\cdot \mid x_{2}, x_{3}\right), p_{1}^{2}\left(\cdot \mid \tilde{x}_{2}, \tilde{x}_{3}\right)\right): x_{2} \cup x_{3} \in U_{\infty}^{N},\right. \\
\left.\tilde{x}_{2} \cup \tilde{x}_{3} \in U_{\infty}^{N}, \quad x_{j}, \tilde{x}_{j} \in X_{j} \quad \text { for } \quad j=1,2\right\},
\end{gathered}
$$

$$
\begin{gathered}
\bar{\alpha}=\sup \left\{\varrho \left(p_{1}^{1}\left(\cdot \mid x_{2}, x_{3}\right), p_{1}^{2}\left(\cdot \mid x_{2}, \tilde{x}_{3}\right): x_{2} \in X_{2}, x_{3}, \tilde{x}_{3} \in X_{3},\right.\right. \\
\text { and } \left.x_{2} \cup x_{3} \in U_{\infty}^{N}, x_{2} \cup \tilde{x}_{3} \in U_{\infty}^{N}\right\} .
\end{gathered}
$$

Lemma 2.2. Let $\beta, z>0$ and an integer $N \geqq 2$ be given. Let a potential $V$ satisfy Condition 1.1 with range less than $c-b$, and let $J$ be an interval on the real line of length $c-b$. Then

$$
\sup _{\substack{s \in U^{N} \\ s \cap J=\emptyset}} \int_{X(J)} \exp \left[-\beta V^{N}(x \mid s)\right] v_{J}(d x)<\infty
$$

Proof. From the proof of Theorem 3.1 of [3], we have $V^{N}(x \mid s) \geqq-D|x|$ for all $x \in X(J)$ and $s \in U_{\infty}^{N}$ such that $s \cap J=\emptyset$, where $D>0$ depends only on $c-b$ and $N$. The proof now follows from the definition of the measure $v_{J}$ given in (1.2).

Lemma 2.3. With the same hypotheses as in Lemma 2.2 and with the identifications given by (2.9) through (2.16),

a) $\bar{\alpha}=0$.

b) $\alpha \leqq 1-h$, for some $h>0$ depending only on $c-b, V^{N}, \beta, z$ (and not $s_{1}$ or $s_{2}$ ).

c) $\varrho\left(r_{[-n, n]}^{(b, c]}\left(\cdot \mid s_{1}\right), r_{[-n, n]}^{(b, c]}\left(\cdot \mid s_{2}\right)\right) \leqq(1-h) \varrho\left(r_{[-n, n]}^{(a, b]}\left(\cdot \mid s_{1}\right), r_{[-n, n]}^{(a, b]}\left(\cdot \mid s_{2}\right)\right)$, where $h$ is given in part $\mathrm{b})$.

Proof. $\bar{\alpha}=0$ follows immediately from the fact that the range of $V^{N}$ is less than $c-b$.

From Lemma 2.2 we see that

$$
\sup _{\substack{s \in U_{N}^{N} \\ s \cap J=\emptyset}} Z_{J}^{N}(s)<\infty
$$

where $Z_{J}^{N}(s)$ is the normalizing constant in (1.5) corresponding to $V^{N}$ and $J$. Let $\emptyset_{J}$ denote the empty configuration in the interval $J$. Since $\exp \left[-\beta V^{N}\left(\emptyset_{J} \mid s\right)\right]$ $=\exp 0=1$, we have

$$
\inf _{t \in U_{\infty}^{N}} \pi_{J}^{N}\left(\left\{\emptyset_{J}\right\}, t\right) \equiv h>0
$$

By consistency of the specification $\left\{\pi_{\Lambda}^{N}\right\}$ (see [5]), for any interval $I \supset J$,

$$
\pi_{I}^{N}\left(\left\{\emptyset_{J}\right\}, s\right)=\int \pi_{J}^{N}\left(\left\{\emptyset_{J}\right\}, t\right) \pi_{I}^{N}(d t, s) .
$$


From (1.7) and since $v_{J}(\{\emptyset\})=1$, it follows that $\pi_{I}\left(\left\{\emptyset_{J}\right\}, t\right)=r_{I}^{J}\left(\emptyset_{J} \mid t\right)$, and thus from (2.18) and (2.19),

$$
\inf _{s \in U_{\infty}^{N}} r_{I}^{J}\left(\emptyset_{J} \mid s\right) \geqq h>0 .
$$

Now combining (2.3), (2.13), and (2.15), we obtain

$$
\alpha=1-\inf \int \min \left[p_{1}^{1}\left(x_{1} \mid x_{2}, x_{3}\right), p_{1}^{2}\left(x_{1} \mid \tilde{x}_{2}, \tilde{x}_{3}\right)\right] v_{J}\left(d x_{1}\right),
$$

where the infimum is taken over the same set of $x_{j}, \tilde{x}_{j}$ 's as in (2.15). Combining this with (2.20) gives $\alpha \leqq 1-h$.

The proof of part c) of the lemma follows directly from parts a) and b), Lemma 3.1, and Remark 3.1. This concludes the proof.

Theorem 2.1. Let $V$ satisfy Condition 1.1. For any $\beta, z>0$, and any integer $N \geqq 2$, there is exactly one Gibbs state for $V^{N}, \beta, z$.

Proof. We first observe that a simple induction argument, together with conclusion c) of Lemma 2.3, shows that for any integer $k>0$,

$$
\varrho\left(r_{[-n, n]}^{(b, c]}\left(\cdot \mid s_{1}\right), r_{[-n, n]}^{(b, c]}\left(\cdot \mid s_{2}\right)\right) \leqq(1-h)^{k}
$$

for all $n$ sufficiently large.

Now given any $s, t \in U_{\infty}^{N}$, let $s_{1} \in U_{\infty}^{N}$ be chosen so that $s_{1} \cap(c, \infty)=s$, and $s_{1} \cap(-\infty, b]=t$. By the triangle inequality,

$$
\begin{aligned}
\varrho\left(r_{[-n, n]}^{(b, c]}(\cdot \mid s), r_{[-n, n]}^{(b, c]}(\cdot \mid t)\right) \leqq & \varrho\left(r_{[-n, n]}^{(b, c]}(\cdot \mid s), r_{[-n, n]}^{(b, c]}\left(\cdot \mid s_{1}\right)\right) \\
& +\varrho\left(r_{[-n, n]}^{(b, c]}\left(\cdot \mid s_{1}\right), r_{[-n, n]}^{(b, c]}(\cdot \mid t)\right) .
\end{aligned}
$$

Given any integer $k>0$, we can conclude from (2.21) that the first term on the right side of (2.22) is bounded by $(1-h)^{k}$ for large enough $n$. Using the relation proved in obvious analogy with (2.21), we can conclude that the second term on the right side of $(2.22)$ is also bounded by $(1-h)^{k}$ for sufficiently large $n$. Since $h$ is independent of $s, t \in U_{\infty}^{N}$, it follows that

$$
\lim _{n \rightarrow \infty} \sup _{s, t \in U_{\infty}^{N}} \varrho\left(r_{[-n, n]}^{(b, c]}(\cdot \mid s), r_{[-n, n]}^{(b, c]}(\cdot \mid t)\right)=0 .
$$

Applying the remarks preceding (2.5) and (2.1) we obtain the conclusion of the theorem.

The following cluster property is analogous to Dobrushin's result $[1$, Corollary 3].

Corollary 2.1. With the same notation and assumptions as in Theorem 2.1, let $D \in B_{[k, \infty)}$ and $C \in B_{(-\infty, k)}$ for some $k>0$. Then

$$
\left|\sigma_{N}(C \cap D)-\sigma_{N}(C) \sigma_{N}(D)\right| \leqq \sigma_{N}(C) F(k),
$$

where $\lim _{k \rightarrow \infty} F(k)=0$.

Proof. From general measure theory it suffices to prove the upper bound in (2.23) for all $D \in B_{[k, m]}$ and all $m$ such that $k<m<\infty$. Since the specification $\left\{\pi_{A}^{N}\right\}$ can be regarded as a system of conditional probabilities for $\sigma_{N}$ (see [5]) we have

$$
\sigma_{N}(D)=\int \pi_{[-k, m]}^{N}(D, s) \sigma_{N}(d s)
$$


and

Consequently,

$$
\sigma_{N}(C \cap D)=\int_{C} \pi_{[-k, m]}^{N}(D, s) \sigma_{N}(d s)
$$

$$
\left|\sigma_{N}(C \cap D)=\sigma_{N}(C) \sigma_{N}(D)\right| \leqq \sigma_{N}(C) \sup _{s, t \in U_{\infty}^{N}}\left|\pi_{[-k, m]}^{N}(D, s)-\pi_{[-k, m]}^{N}(D, t)\right| .
$$

Since $\pi_{[-k, m]}^{N}(D, s)=\int \pi_{[-k, k]}^{N}(D, t) \pi_{[-k, m]}^{N}(d t, s)$, it follows that

$$
\left|\sigma_{N}(C \cap D)-\sigma_{N}(C) \sigma_{N}(D)\right| \leqq \sigma_{N}(C) \sup _{s, t \in U_{\infty}^{N}}\left|\pi_{[-k, k]}^{N}(D, s)-\pi_{[-k, k]}^{N}(D, t)\right| .
$$

Let $F(k)=\sup _{s, t \in U_{\infty}}\left|\pi_{[-k, k]}^{N}(D, s)-\pi_{[-k, k]}^{N}(D, t)\right|$. From the proof of Theorem 2.1 it follows that

This completes the proof.

$$
\lim _{k \rightarrow \infty} F(k)=0
$$

\section{Nonhard-Core Case}

Existence for all $\beta, z>0$ of a tempered Gibbs state for a potential satisfying Condition 1.1 follows from results in [4]. In this section we prove the uniqueness for all $\beta, z>0$ of the tempered Gibbs state for such potentials. This is accomplished by proving the existence of precisely one extremal tempered Gibbs state corresponding to $V, \beta, z$. The technique of proof relies on Theorem 2.1 , the methods developed in [4], and the general theory of Gibbs states as developed in $[5]$.

The proof of Theorem 2.2 of [5] shows that any extremal tempered Gibbs state $\sigma^{s}$ for $V, \beta, z$ is obtained via the limit,

$$
\sigma^{s}(A)=\lim _{n \rightarrow \infty} \pi_{[-n, n]}(A, s)
$$

for some $s \in U_{\infty}$ and any cylinder set $A \in B_{\mathbb{R}}$. It also follows from Theorem 2.2 of [5] that for any Gibbs state $\sigma$ corresponding to $V, \beta, z$ and any $A \in B_{\mathbb{R}}$,

$$
\sigma(A)=\int_{U_{\infty}} \sigma^{s}(A) \sigma(d s)
$$

where $\sigma^{s}$ is an extremal tempered Gibbs state for each $s \in U_{\infty}$ and is given by (3.1) for all $s$ in some subset of $U_{\infty}$ of $\sigma$-measure one (see [5] for further details). We see from (3.2) that the existence of one and only one extremal tempered Gibbs state implies the existence of one and only one tempered Gibbs state.

Let a potential $V$ satisfying Condition 1.1 be given and recall from Definition 1.1 that $V^{N}=V+\varphi_{N}$. It is easily checked that (see Definition 1.2)

$$
U_{\infty}=\bigcup_{N \geqq 2} U_{\infty}^{N} \text {. }
$$

Now given $s \in U_{\infty}$ and $\beta, z>0$, suppose that $\sigma^{s}$ is a tempered extremal Gibbs state for $V, \beta, z$ and is given by (3.1). From Theorem 2.2 of [4] and (3.3) above it follows that

$$
\sigma_{N}^{s}(A)=\lim _{n \rightarrow \infty} \pi_{[-n, n]}^{N}(A, s)
$$


for all $N$ sufficiently large and any cylinder set $A \in B_{\mathbb{R}}$, where $\sigma_{N}^{s}$ is an extremal Gibbs state for $V^{N}, \beta, z$. We show in Theorem 3.1 below that for appropriate $s \in U_{\infty}$,

$$
\lim _{N \rightarrow \infty} \sigma_{N}^{s}(A)=\sigma^{s}(A)
$$

for any cylinder set $A \in B_{\mathbb{R}}$.

Lemma 3.1. Let $\beta, z>0$ and a potential $V$ satisfying Condition 1.1 be given. Let $\sigma$ be any tempered Gibbs state for $V, \beta, z$. Then for any $\delta>0$,

$$
\sigma\left\{t \in U_{\infty}: \pi_{[-n, n]}\left(U_{m}, t\right)>1-\delta \text { for some fixed } m \text { and all } n\right\}=1 .
$$

Proof. By hypothesis $\sigma\left(U_{\infty}\right)=1$. Therefore, given $\varepsilon>0$, we can choose $m$ large enough so that $\sigma\left(U_{m}\right)>1-\varepsilon$. Let

$$
\lambda_{n, k}=\sigma\left\{t \in U_{\infty}: \pi_{[-n, n]}\left(U_{m}, t\right)<1-k \varepsilon\right\} .
$$

Since $\pi_{[-n, n]}\left(U_{m}, t\right) \leqq 1$ for all $t \in U_{\infty}$, it follows that

$$
1-\varepsilon<\sigma\left(U_{m}\right)=\sigma\left(\pi_{[-n, n]}\left(U_{m}, t\right)\right)<1-\lambda_{n, k}+\lambda_{n, k}(1-k \varepsilon)
$$

or $1-\varepsilon<1-\lambda_{n, k} k \varepsilon$, and consequently $\lambda_{n, k}<\frac{1}{k}$. We have shown that

$$
\sigma\left\{t \in U_{\infty}: \pi_{[-n, n]}\left(U_{m}, t\right)<1-k \varepsilon\right\}<\frac{1}{k}
$$

for all $n=1,2,3, \ldots$. Replacing $k \varepsilon$ by $\delta$ in (3.5), we see that given any $\delta>0$ and any integer $k \geqq 1$, there exists an $m \geqq 1$ such that

$$
\pi_{[-n, n]}\left(U_{m}, t\right)>1-\delta
$$

for all $n \geqq 1$ and all $t \in U_{\infty}$ in a set of $\sigma$-measure greater than $1-1 / k$. The conclusion of Lemma 3.1 now follows from the fact that $k$ can be chosen arbitrarily large.

Remark 3.1. Lemma 3.1 is valid for higher dimensions.

Theorem 3.1. Let $\sigma$ be an extremal tempered Gibbs state for $V, \beta, z$ and assume $V$ satisfies Condition 1.1. Then there exists an $s \in U_{\infty}$ such that $\sigma=\sigma^{s}$ and such that for any cylinder set $A \in B_{\mathbb{R}}$,

$$
\lim _{N \rightarrow \infty} \sigma_{N}^{s}(A)=\sigma^{s}(A),
$$

where $\sigma^{s}$ and $\sigma_{N}^{s}$ are given by (3.1) and (3.4), respectively.

Proof. From Theorem 2.2 of [5] and Lemma 3.1 above it follows that there exists an $s \in U_{\infty}$ satisfying: 1) $\sigma=\sigma^{s}$, where $\sigma^{s}$ is given by $(3.1), 2$ ) given any $\varepsilon>0$, there exists $m \geqq 1$ such that

$$
\pi_{[-n, n]}\left(U_{m}, s\right)>1-\varepsilon
$$

uniformly in $n$. With (3.7) the proof of Lemma 3.1 of [4] yields the following. Given any $\varepsilon>0$ and any cylinder set $A \in B_{\mathbb{R}}$,

$$
\left|\pi_{[-n, n]}^{N}(A, s)-\pi_{[-n, n]}(A, s)\right|<\varepsilon
$$


for all $N$ sufficiently large uniformly in $n$. By the triangle inequality,

$$
\begin{aligned}
& \left|\sigma_{N}^{s}(A)-\sigma^{s}(A)\right| \leqq\left|\sigma_{N}(A)-\pi_{[-n, n]}^{N}(A, s)\right| \\
& \quad+\left|\pi_{[-n, n]}^{N}(A, s)-\pi_{[-n, n]}(A, s)\right|+\left|\pi_{[-n, n]}(A, s)-\sigma^{s}(A)\right| .
\end{aligned}
$$

Combining (3.1), (3.4), (3.8), and (3.9) and first choosing $N$ and then $n$ large, we see that the right side of (3.9) can be made arbitrarily small and thus

This concludes the proof.

$$
\lim _{N \rightarrow \infty} \sigma_{N}^{s}(A)=\sigma^{s}(A) \text {. }
$$

Remark 3.2. Theorem 3.1 holds for arbitrary dimension provided Lemma 3.1 of [4] holds for arbitrary dimension. In contrast to the statement of Lemma 3.1 of [4], the proof, as given in [4] is valid only for one-dimensional systems.

Theorem 3.2. Let $V$ satisfy Condition 1.1. For any inverse temperature $\beta$ and chemical activity $z$, there is exactly one tempered Gibbs state $\sigma$ corresponding to $V, \beta, z$. Furthermore,

$$
\lim _{N \rightarrow \infty} \sigma_{N}(A)=\sigma(A)
$$

for any cylinder set $A \in B_{\mathbb{R}}$, where $\sigma_{N}$ is the unique Gibbs state for $V^{N}, \beta$, z given by Theorem 2.1.

Proof. From Theorem 3.1 and the remarks preceding (3.4) it follows that for any $s, t \in U_{\infty}, \sigma_{N}^{t}=\sigma_{N}^{s}$ for all $N$ sufficiently large. From Theorem 3.1 it follows that all extremal tempered Gibbs states for $V, \beta, z$ are identical and consequently from (3.2) there exists precisely one tempered Gibbs state $\sigma$ for $V, \beta, z ; \sigma$ is clearly given by (3.10). This concludes the proof.

Remark 3.3. The results on the decay of correlations given in [4] hold for the potentials satisfying Condition 1.1 considered here.

\section{References}

1. Dobrushin, R.L.: Conditions for the absence of phase transitions in one-dimensional classical systems. Math. Sbornik 93, 29-49 (1974)

2. Campanino, M., Capocaccia, D., Olivieri, E.: Analyticity for one-dimensional systems with long range superstable interactions. J. Stat. Phys. 33, 437-476 (1983)

3. Klein, D.: Dobrushin uniqueness techniques and the decay of correlations in continuum statistical mechanics. Commun. Math. Phys. 86, 227-246 (1982)

4. Klein, D.: Convergence of grand canonical gibbs measures. Commun. Math. Phys. 92, 295-308 (1984)

5. Preston, C.: Random fields. In: Lecture Notes in Mathematics, Vol. 534. Berlin, Heidelberg, New York: Springer 1976

6. Ruelle, D.: Superstable interactions in classical statistical mechanics. Commun. Math.Phys. 18, 127-159 (1970)

7. Suhov, Yu.: Random point processes and DLR equations. Commun. Math. Phys. 50, 113-132 (1976)

Communicated by J. Fröhlich

Received July 18, 1984 